\title{
Structural Studies on the Radio-Protective Effect of Lycopene (Tomato Supplementation) Against Hepatic Cellular Injury Induced by Chronic Doses of Gamma Radiation
}

\author{
Hanaa Fathy Waer ${ }^{1 *}$ and Fatma Mohsen Shalaby ${ }^{2}$ \\ ${ }^{1}$ Atomic Energy Authority, National Center for Radiation Research and Technology, Egypt \\ ${ }^{2}$ Zoology department, Faculty of Sciences, Mansoura University, Egypt
}

\begin{abstract}
Background and aim: Antioxidants are substances that may protect our bodies against the effects of freeradicals. Free radicals are molecules produced when our body breaks down food, or by environmental exposures like radiation. Free radicals can damage cells, our goal of this work is to study the damage resulted by chronic low doses of gamma radiation using natural antioxidant like Lycopene that found in tomato that are available to most of the people especially those that work in the field of radiation.
\end{abstract}

Materials and methods: The radio protective effect of Lycopene against liver damage was investigated in 40 male albino rats divided into 4 groups (10 per group). Group 1 rats considered normal controls, group 2, considered irradiated group which were exposed to fractionated doses of $1 / 2 \mathrm{~Gy}$ every two days for one month, group3, considered control group which delivered Lycopene alone (5 mg/kg per day) for two months, the last group was group 4 which considered irradiated animals given Lycopene for two months at the same dose of group 2 started from the first day of experiment and extent for to last day. Animals of groups 1 and 2 were sacrificed after one month while groups 3 and 4 were sacrificed after 2 months. Samples were taken and prepared for microscopic studies.

Results: Examined sections showed that there were no cellular changes in group 2, while a significant cellular injury was clearly observed in group 3 on the other hand. Group4 showed significant decrease in the pathological changes occurred in compared with group 3 .

Conclusion: We come to the fact that Lycopene supplementation may be helpful to reduced pathological cellular injuries in the liver cells induced by accumulated doses of radiation exposure further studies may be done to confirm these findings.

Keywords: Lycopene; Radiation; Rat; Antioxidant; Liver injury; Histopathology; Cytology

\section{Introduction}

Biological effects of radiation results mainly from oxidative stress that leads to both DNA damage and cell death $[1,2]$. When cells are exposed to ionizing radiation the standard physical effects between radiations and the atoms or molecules of the cells occur first and biological damage to cell functions follows later [3]. Radiation exposure alters the balance of endogenous defense system through generation of ROS resulting in an imbalance in prooxidant, antioxidant status in the cell $[4,5]$. The degree of protection against $\gamma$-radiation injuries depends on many complex factors such as dosage, time and distance [6]. Natural antioxidants play a recent source of protection against $\gamma$-radiation. Antioxidants may protect cells from the damage caused by unstable molecules known as free radicals, which involved in several disorders [7].

Appropriate antioxidant may inhibit or reduce free radical toxicity and offer protection against radiation damage. A number of dietary antioxidants have been reported to decrease free radical attack on biomolecules like Lycopene [8]. Which is a dietary carotenoid synthesized by plants and microorganisms. It occurs primarily in red fruits, vegetables and tomatoes [9]. Lycopene was found to exhibited local anti-inflammatory activity, attenuated liver injury and suppress liver cancer $[10,11]$.

The present study aims to evaluate the beneficial effect of lycopene, which is a relatively carotenoid found mainly in tomato against pathological changes induced by accumulated doses of $\gamma$-radiation.

\section{Materials and Methods}

The present study was done on healthy adult male albino rats in the weight range from (150-200 gm), selected from an inbred group housed in specially designed cages and maintained under standard conditions of light and temperature all animals were cared for according to the Guiding Principle in the Care and Use of Animals. They were divided in to 4 groups each of 10 rats on the basis of initial weight and kept in individual cages. Group 1: considered normal Control, group 2 considered irradiated animals exposed to fractionated doses of gamma radiation, (1/2Gy ) every two days for one month. Group 3: considered control animals delivered Lycopene alone orally at a dose of $(5 \mathrm{mg} / \mathrm{kg}$ per day) for two months. The last group was group 4 which considered irradiated animals given Lycopene for two months at the same dose of group 2 started from the first day of experiment and extent for to last

*Corresponding author: Hanaa F. Waer, Professor of Cell Biology, National Center for Radiation Research and Technology, 87 Abdel-shafy Mohamed, Hay El Zohor, Madinet Nasr, Cairo, Egypt, Tel: 00201006950192, 0966563535597; E-mail: hanaawaer@yahoo.com

Received March 21, 2012; Accepted June 18, 2012; Published June 20, 2012

Citation: Waer HF, Shalaby FM (2012) Structural Studies on the RadioProtective Effect of Lycopene (Tomato Supplementation) Against Hepatic Cellular Injury Induced by Chronic Doses of Gamma Radiation. J Cytol Histol 3:145 doi:10.4172/2157-7099.1000145

Copyright: @ 2012 Waer HF, et al. This is an open-access article distributed under the terms of the Creative Commons Attribution License, which permits unrestricted use, distribution, and reproduction in any medium, provided the original author and source are credited. 
Citation: Waer HF, Shalaby FM (2012) Structural Studies on the Radio-Protective Effect of Lycopene (Tomato Supplementation) Against Hepatic Cellular Injury Induced by Chronic Doses of Gamma Radiation. J Cytol Histol 3:145. doi:10.4172/2157-7099.1000145

day. Animals of groups 1 and 2 were sacrificed after one month while groups 3 and 4 were sacrificed after 2 months. Samples were taken and prepared for microscopic studies.

\section{Natural antioxidants}

Lycopene Extract Liquid Alcohol Free was purchased from Sigma Chemical Co. and used at dose ( $5 \mathrm{mg} / \mathrm{kg}$ per day).

\section{Radiation facility}

Fractionated dose of $0.5 \mathrm{~Gy}$ gamma irradiation was performed at the National Centre for Radiation Research and Technology, Atomic Energy Authority (NCRRT), Cairo, Egypt, using Cesium - 137 in a Gamma cell-40 Irradiator (Atomic Energy of Canada Limited, Canada). Animals were exposed to radiation every 2 days, at a dose rate of 0.61 Gy min-1 for one month.

\section{Light microscopy}

Liver tissue biopsy was immediately taken and cut into pieces of desired size and fixed in Bouin's fixative (saturated picric acid $75 \mathrm{ml}+$ conc. Formalin $25 \mathrm{ml}+$ glacial acetic acid $5 \mathrm{ml}$ ) for $24-48 \mathrm{hrs}$. Tissues were removed from fixative solution and washed thoroughly with distilled water for few hours so as to remove extra Bouin's fixative. After washing, samples were washed with $70 \%$ alcohol to remove excess of picric acid from the tissues and dehydrated in graded series of ethanol $5 \mu \mathrm{m}$ thick histological sections were cut and stained with Hematoxylin and eosin and examined under bright field light microscope [12].

\section{Electron microscopic investigation}

Liver were removed from the anesthetized animals and killed by decapitation, sliced into one $\mathrm{mm}$ pieces in a drop of $3 \%$ glutareldehyde. Tissue was then immersed in fresh ice cold fixative for two hours and then in $0.1 \mathrm{M}$ cacodylate buffer for next $4 \mathrm{hrs}$ and post fixed in $1 \%$ osmic acid for one to two hours. Liver sections was then dehydrated in an ascending series of alcohol, followed by propylene oxide and finally embedded in araldite resin that was polymerized at $60^{\circ} \mathrm{C}$. Selected blocks were cut with a diamond knife, picked up on copper grids and stained with uranyl acetate and lead citrate for final viewing [13].

\section{Results}

\section{Light microscopy}

Examined liver sections of Group 1which considered normal control animals showed normal pattern of cellular structures (Figure 1), meanwhile examined sections of Group 2 which was exposed to accumulated dose of $0.5 \mathrm{~Gy}$ of $\gamma$-radiation every 2 days for one month showed sever changes the cytoplasm was disintegrated and vacuolated, area of necroses, pyknosis, fatty change and inflammation could be seen (Figures 2,3). Atrophy, hemorrhage, apoptosis, pyknosis and hyperplasia are common features (Figures 4,5). Examined sections from Group 3 which delivered Lycopene alone showed no remarkable pathological changes (Figure 6). On the other hand examined section from group 4 which was supplemented by Lycopene as radio protectors, showed a trend toward lowering incidence of hepatic pathological changes induced by $\gamma$-radiation, a remarkable restoration of normal cell structure. The cells retain its regularity and sizes, normal homogenous cytoplasm with rounded nucleus (Figures $7 \mathrm{a}$ and $7 \mathrm{~b}$ ).

\section{Electron microscopic investigation}

Fine structure examination of group1 which considered normal control showed normal pattern of cellular structures, healthy nucleus,

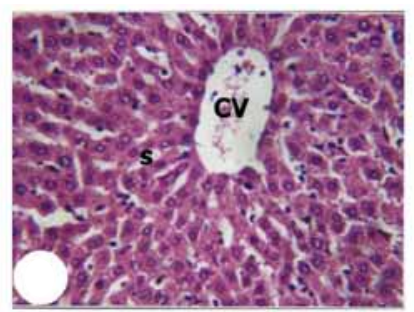

Figure 1: Light micrograph of a section from normal rat liver (Group1) showing radial organization of hepatocytes and sinusoids $(\mathbf{S})$ around the central vein $(\mathbf{C V})$. [H\&E] 100x

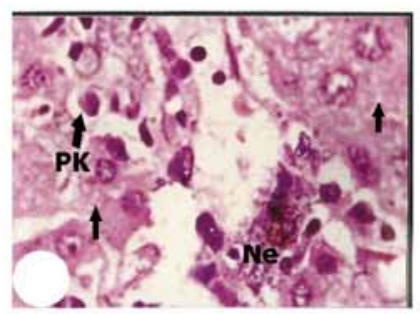

Figure 2: light micrograph of a section from rat liver exposed to accumulated dose of gamma radiation (Group 2) presented gradual vacuolization (arrows), cytoplasmic disintegration, Pyknotic Nuclei (PK) Hepatic Necrosis (Ne), inflammation and fatty accumulation [H\&E] 200x.

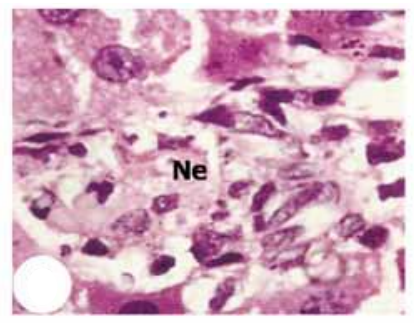

Figure 3: Light micrograph of a section rat liver expose $H \& E$ d to accumulated dose of gamma radiation (Group2) presented, Area of Necrosis (Ne). [H\&E] 400x.

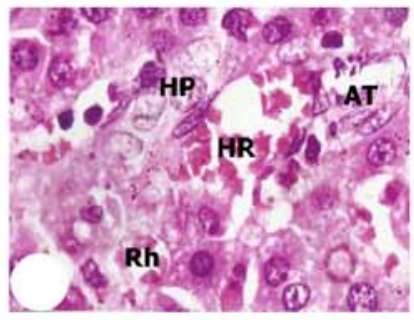

Figure 4: Light micrograph of a section of rat liver exposed to accumulated dose of gamma radiation (Group 2) presented Hemorrhage (HR), Globular Red Hyaline (Rh), Atrophy (AT) and Hyperplasia (Hp). [H\&E] 400x.

with homogeneous cytoplasm, healthy mitochondria with double membrane .The rough endoplasmic reticulum was well designed with ribosome and few glycogen rosette forms were observed (Figures 8 and 9).

Mean while examined sections from (Group 2) which exposed to accumulated dose of gamma radiation revealed an obvious pathological 
Citation: Waer HF, Shalaby FM (2012) Structural Studies on the Radio-Protective Effect of Lycopene (Tomato Supplementation) Against Hepatic Cellular Injury Induced by Chronic Doses of Gamma Radiation. J Cytol Histol 3:145. doi:10.4172/2157-7099.1000145

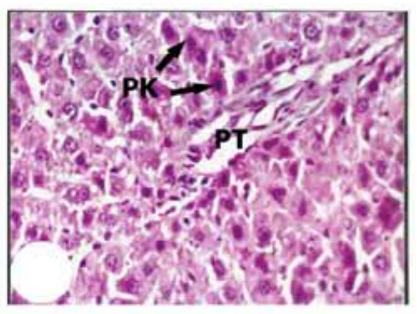

Figure 5: Light micrograph of a section rat liver exposed to accumulated dose of gamma radiation (Group 2) presented Pyknosis (PK) and congestion of the Portal Track (PT). [H\&E] 200x.

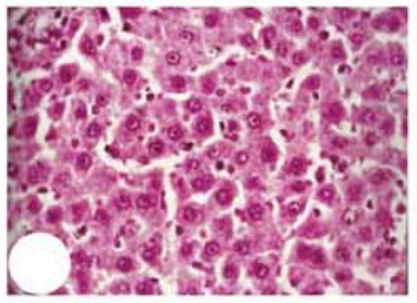

Figure 6: Light micrograph of a section of rat liver cell given Lycopene alone ( $5 \mathrm{mg} / \mathrm{kg}$ per day) (Group 3 ) showing no restricted cellular changes. [H\&E] $200 x$.
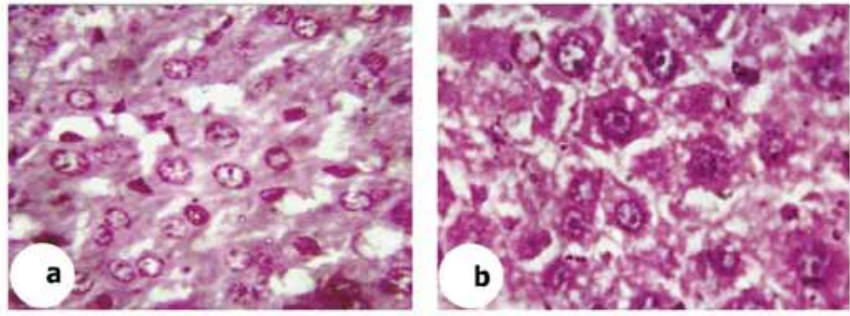

Figure 7 ab: Light micrograph of a section of gamma irradiated rat liver given Lycopene (5 mg/kg per day) (Group 4) showing great amelioration and recovery of the hepatic cells. Lipid and fatty features are still recorded. [H\&E] 400x.

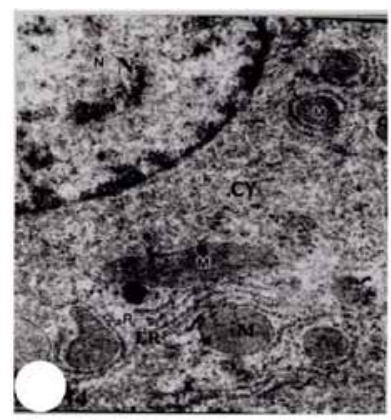

Figure 8: An electron micrograph of a section of normal control liver rat (Group1) showing normal structure illustrated in healthy nucleus $(\mathbf{N})$, homogeneous cytoplasm (Cy) with well developed mitochondria (M) and rough endoplasmic reticulum (RER). [H\&E] 6000x

cell changes. The cytoplasm lost its normal organization, contained some necrotic areas and many lipid vacuoles (lipoma). Amorphous polymorphic mitochondria were found to be malformed, in some hepatocytes like degradation the outer mitochondrial membrane (Figure 10). Rough endoplasmic reticulum was seen as elongated ruptured and fragmented with detachment of ribosome. Collagen fiber was also noticed in many areas of cytoplasm as a feature of liver fibroses (Figure 13). Also, the nucleus showed many morphological alterations like irregular and dilated envelope, and obvious clumping of heterochromatin (Figures 11 and 12).

Liver sections from group 3 which delivered only Lycopene showed no remarkable cellular changes (Figures 14-15). From the other side of view those examined from (Group 4) which was treated by Lycopene showed an ameliorant sign of Lycopene, The nucleus retains its normality, semi-rounded and contained permanent, heterochromatin tightly packed form of chromatin (Figures 16 and 17). The cytoplasm was homogeneous and granulated with well designed strands of

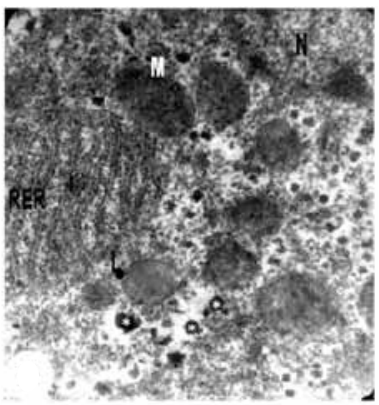

Figure 9: An electron micrograph of a section of normal control rat liver (Group1) showing parallel strands of rough endoplasmic reticulum (RER) with ribosome . Rosette form of glycogen particles (white stars) 6000x.

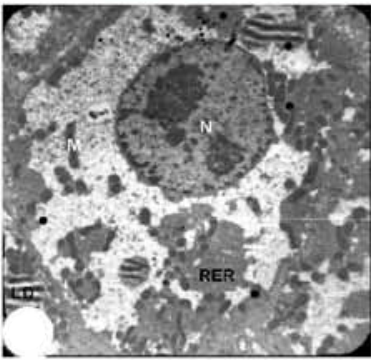

Figure 10: An electron micrograph of a section of irradiated rat liver (Group2) showing markedly altered morphology. The nucleus showed irregular nucleus $(\mathrm{N})$ and reduced number of mitochondria $(\mathrm{M})$ ), destructive (RER),lipid droplets (LD) . 4000x.

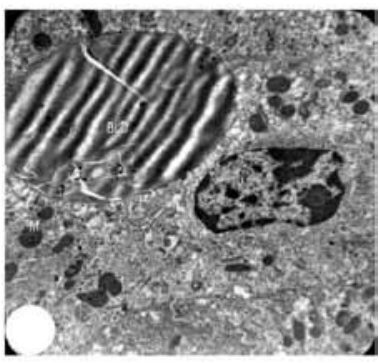

Figure 11: an electron micrograph of a section of irradiated rat liver (Group 2). Note, lipoma can be restricted in a big lipid droplet ( BLD ). 6000x. 
Citation: Waer HF, Shalaby FM (2012) Structural Studies on the Radio-Protective Effect of Lycopene (Tomato Supplementation) Against Hepatic Cellular Injury Induced by Chronic Doses of Gamma Radiation. J Cytol Histol 3:145. doi:10.4172/2157-7099.1000145

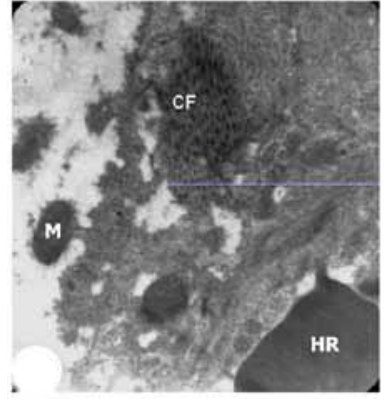

Figure 12: an electron micrograph of a section of irradiated rat liver (Group 2) showing hemorrhage (HR ), increased amount of collagen fiber (CF ) and malformated mitochondria (M). 8000x.

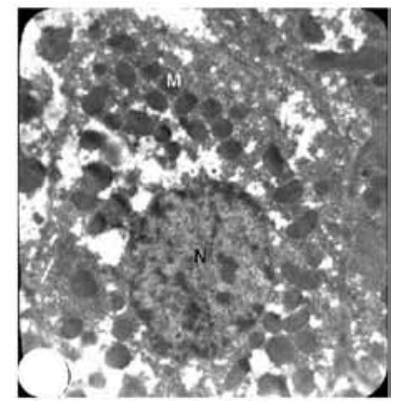

Figure 13: An electron micrograph of a section of irradiated rat liver (Group2) showing several striking alterations of mitochondrial ultra structure. $4000 x$.

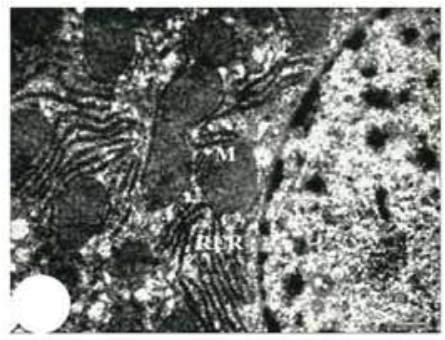

Figure 14: An electron micrograph of a section of rat liver given Lycopene alone (5 mg/kg per day) (Group 3) for Two months showing no cellular changes, the Mitochondria (M) and the rough endoplasmic reticulum (RER) showed no cellular changes . $8000 x$.

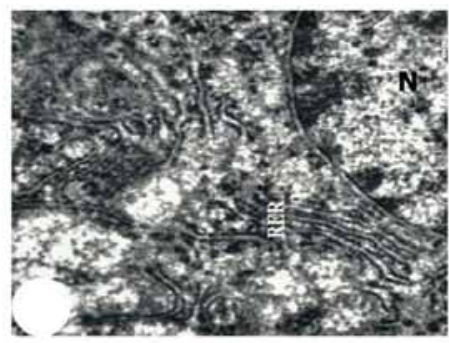

Figure 15: An electron micrograph of a section of rat liver given Lycopene alone (5 mg/kg per day) (Group3) for Two months ,Note classic cell structure with healthy nucleus (N), and endoplasmic reticulum with ribosome attached (RER). 8000x. rough Endoplasmic Reticulum (rER) and ribosome (Figure 18). On the other hand further examined sections revealed that lipid droplets still recognized in the cell but not as in the treated sections. Also some sections shows that the cytoplasm still vacuolated (Figure 19 and 20).

\section{Discussion}

Ionizing radiation is known to induce oxidative stress through generation of ROS resulting in an imbalance in prooxidant, antioxidant status in the cells [14]. When radiation is absorbed by a living cell, the primary damage is caused by ionization and excitation of the atoms and molecules of that cell resulting in changes in cellular structure, damage to essential constituents, and observable biological injury [15].

Light microscopic observations in the present study showed much cellular destruction and several pathological changes like conspicuous disorganization of the nucleus including morphological alterations in irradiated animals. This is could be explained by that radiation

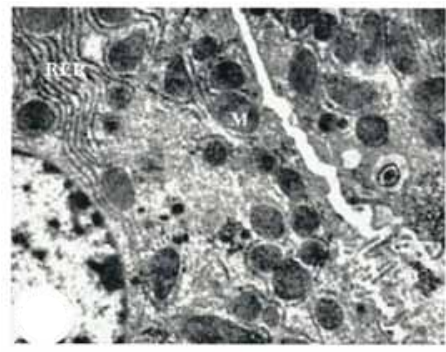

Figure 16: An electron micrograph of a section of liver from Group 4 which was treated with Lycopene and radiation. A significant regression in the rate of the degenerative changes was found 6000x.

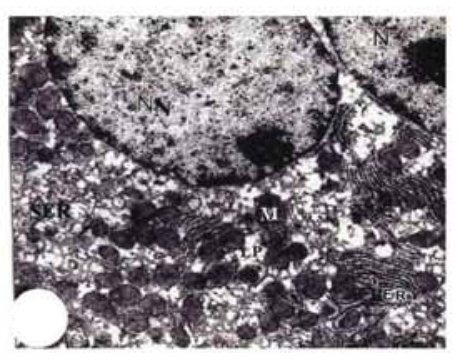

Figure 17: An electron micrograph of a section of liver from Group 4 which was treated with Lycopene and radiation showing cytoplasm with normal patches of smooth and rough endoplasmic reticulum and ribosomes inside. The nucleus( $\mathbf{N}$ )looks more healthy with euchromatin. 8000x.

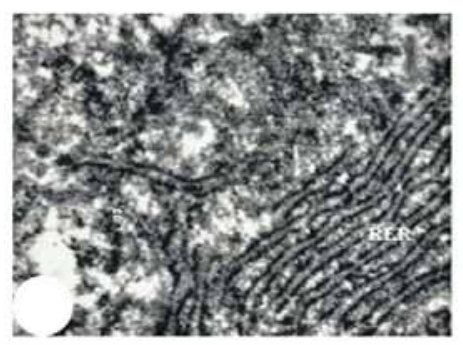

Figure 18: An electron micrograph of a section of liver from Group 4 which was treated with Lycopene and radiation showing nearly normal structure. $10000 x$. 
Citation: Waer HF, Shalaby FM (2012) Structural Studies on the Radio-Protective Effect of Lycopene (Tomato Supplementation) Against Hepatic Cellular Injury Induced by Chronic Doses of Gamma Radiation. J Cytol Histol 3:145. doi:10.4172/2157-7099.1000145

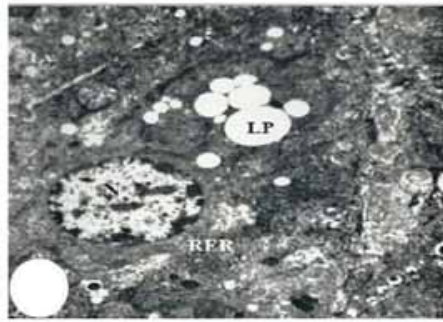

Figure 19: An electron micrograph of a section of liver from Group 4 which was treated with Lycopene and radiation Note that lipid droplets still found in abundant (Lp). 4000x.

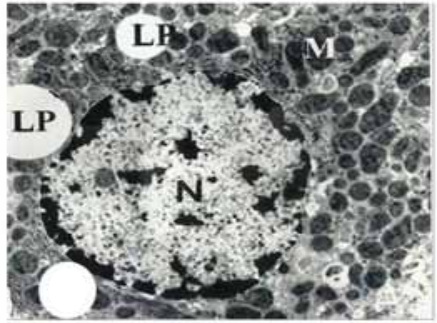

Figure 20: Higher magnification of Figure 19 Note increased amount of lipid droplets. $6000 x$.

is a well-known inducer of free radicals caused to chromosomal damages [16]. The cytoplasm was ill defined and lost its normality containing necrotic areas and pyknosis. Disorganization of the endoplasmic reticulum and malformed, shrieked mitochondria were also recognized. This is may attributed to that oxidative stress induced by radiation through generation of ROS that damages proteins, lipids and nucleic acids [17]. Exposure to $\gamma$-irradiation inducing organelle dysfunction induced by cytochrome P450 E1 (CYP2E1) in the liver, which might be associated with mitochondrial damage $[18,19]$. Also radiation may affect mitochondrial protein complexes. The present results also showed that Lycopene ameliorate the injuries induced by $\gamma$-radiation. The cells nearly retain its normal structure, regularity, size and normal homogenous central rounded nucleolus. This is may be due to that the use of antioxidants (Lycopene) may help in decreasing the geno-toxicity created by radiation and may inhibit mutagenesis and carcinogenesis [16]. These were in accordance with studies which come to the fact that the imbalance in antioxidant status in the cells as a result of ionizing may be ameliorated as result of administration of Lycopene [20]. Lycopene significantly restored the antioxidant enzymes Superoxide Dismutase (SOD), Glutathione Peroxidase (GSH$\mathrm{Px}$ ), and Glutathione Reductase (GR); Reduced Glutathione (GSH); and decreased levels of the lipid peroxide Malondialdehyde (MDA) in hypertensive patients [21]. Lycopene was found to have a favorable effect in reducing MDA levels and increasing GSH levels in coronary artery disease in postmenopausal women [22]. Lycopene also has been found to inhibit proliferation of several types of cancer cells [23].

Epidemiological studies have suggested that regular consumption of tomatoes, the most common source of lycopene in human diets is associated with a reduced risk of certain forms of cancer and other diseases. This postulated protective effect of Lycopene is thought to be due to the various antioxidant properties of this compound [24].

Electron microscopic investigation in the present study showed that supplementation with Lycopene offers great amelioration in the structure of the cellular organoids against $\gamma$-irradiation induced cellular damage. The cytoplasm retained its granulation. The ER gained its normal pattern with restoration of ribosomes. The nucleus, mitochondria get its normality $[25,26]$. These results run barrel with Srinivasan [21], who showed that pretreatment with Lycopene offers protection to normal lymphocytes against $\gamma$-radiation-induced cellular damage due to free radical released by radiation reaction inside the cell. The ameliorative effect of Lycopene may be resulted from; it acts as a scavenger to the free radicals that causes damage to the cells and tissues. It has been found to be effective against oxidation by atmospheric oxygen (auto oxidation) [27]. Other interesting finding in this study was that lipid droplets still found in Lycopene treated animals in considerable averages compared to $\gamma$ - irradiated rats [28]. This is may be due to the antioxidant sparing action of Lycopene in decreasing lipid peroxidation and improved antioxidant status preventing the damage to the cell [21].

\section{Conclusion}

Lycopene may have considerable therapeutic potential as a cheap natural antioxidant that was available to most people especially those who worked in the field of radiation.

\section{References}

1. Tolman KG, Fonseca V, Tan MH, Dalpiaz A (2004) Narrative review: hepatobiliary disease in type 2 diabetes mellitus. Ann Intern Med 141: 946-956.

2. Steel GG (1996) From targets to genes: a brief history of radio sensitivity. Phys Med Biol 41: 205-222.

3. Hall EJ (2000) A radiation biologist looks to the future. Int J Radiat Oncol Bio Phys 46: 1-2.

4. Samarth RM, Kumar A (2003) Mentha Piperia leaf extract provides protection against radiation induced chromosomal damage in bone marrow of mice. Indian J Exp Biol 41: 229-237.

5. Bhosle SM, Huilgol NG, Mishra KP (2005) Enhancement of radiation induced oxidative stress and cytotoxicity in tumor cells by ellagic acid. Clin Chim Acta 359: 89-100.

6. Vincent E. Giuliano (2008) Protection against radiation-the second line of defense.

7. Gerber M, Boutron-Ruault MC, Hercberg S, Riboli E, Scalbert A, et al. (2002 ) Food and cancer: state of the art about the protective effect of fruits and vegetables. Bull Cancer 89: 293-312.

8. El-Habit OH, Saada HN, Azab KS, Abdel-Rahman M, El-Malah DF (2000) The modifying effect of B-carotene on gamma radiation-induced elevation reactions and genotoxicity in male rat. Mutat Res 466: 179-186.

9. Giovannucci E (1999) Tomato, tomato-based products, lycopene and cancer: review of the epidemiologic literature. J Natl Cancer Inst 91: 317-331.

10. Ralph W. Moss (2003) Lycopene In the Prevention and Treatment of Various Cancers.

11. Bignotto L, Rocha J, Sepodes B, Eduardo-Figueira M, Pinto R, et al. (2009) Anti-inflammatory effect of lycopene on carrageenan-induced paw oedema and hepatic ischaemia-reperfusion in the rat. Br J Nutr 102: 126-133.

12. Gretchen L. Humason (1979) Animal tissue techniques. WH Freema.

13. Palade GE, Bruns RR (1968) Structural modulations of plasmalemmal vesicles J Cell Biol 37: 633-649.

14. Hahn SM Krishna MC, Samuni A, DeGraff W, Cupeta DO, et al. (1994) Potential use of nitroxides in radiation oncology. Cancer Res 54: 2006s-2010s

15. Bhosle SM, Huilgol NG, Mishra KP (2005) Enhancement of radiation-induced oxidative stress and cytotoxicity in tumor cells by ellagic acid. Clin Chim Acta 359: $89-100$

16. Flora SJ, Bhadauria S, Kannan GM, Singh N (2007) Arsenic induced oxidative stress and the role of antioxidant supplementation during chelation: a review. J Environ Biol 28: 333-347. 
Citation: Waer HF, Shalaby FM (2012) Structural Studies on the Radio-Protective Effect of Lycopene (Tomato Supplementation) Against Hepatic Cellular Injury Induced by Chronic Doses of Gamma Radiation. J Cytol Histol 3:145. doi:10.4172/2157-7099.1000145

Page 6 of 6

17. Chevion S, Or R, Berry EM (1999) The antioxidant status of patients subjected to total body irradiation. Biochem Mol Biol Int 47: 1019-1027.

18. Claro S, Oshiro ME, Freymuller E, Katchburian E, Kallas EG, et al. (2008) Gamma-radiation induces apoptosis via sarcoplasmatic reticulum in guinea pig ileum smooth muscle cells. Eur J Pharmacol 590: 20-28.

19. Flora SJ, Mehta A, Gautam P, Jatav PC, Pathak U (2007) Essential metal status, prooxidant/antioxidant effects of MiADMSA in male rats: age-related effects. Biol Trace Elem Res 120: 235-247.

20. Flora SJ, Chouhan S, Kannan GM, Mittal M, Swarnkar H ( 2008) Combined administration of taurine and monoisoamyl DMSA protects arsenic induced oxidative injury in rats. Oxid Med Cell Longev 1: 39-45.

21. Srinivasan M, Devipriya N, Kalpana KB, Menon VP (2009) Lycopene: an antioxidant and radioprotector against gamma-radiation-induced cellular damages in cultured human lymphocytes. Toxicology 262: 43-49.

22. Srinivasan M, Sudheer AR, Pillai KR, Kumar PR, Sudhakaran PR, et al. (2007) Lycopene as a natural protector against $y$-radiation induced DNA damage, lipid peroxidation and antioxidant status in primary culture of isolated rat hepatocytes in vitro. Biochim Biophys Acta 1770: 659-665.
23. Rao AV, Agarwal S (1999) Role of lycopene as antioxidant carotenoid in the prevention of chronic diseases: a review. Nutr Res 19: 305-323.

24. Rao AV, Agarwal S (2000) Role of antioxidant lycopene in cancer and heart disease. J Am Coll Nutr 19: 563-569.

25. Misra R, Mangi S, Joshi S, Mittal S, Gupta SK, et al. (2006) LycoRed as an alternative to hormone replacement therapy in lowering serum lipids and oxidative stress markers: a randomized controlled clinical trial. J Obste Gynaecol Res 32: 299-304.

26. Rana S, Kumar R, Sultana S, Sharma RK (2010) Radiation-induced biomarkers for the detection and assessment of absorbed radiation doses. J Pharm Bioallied Sci 2: 189-196.

27. Mridula (2011) Introduction to our body protectors - the antioxidants and the benefits we get by knowing them.

28. Bose KS, Agrawal BK (2008) Effect of lycopene from cooked tomatoes on serum antioxidant enzymes, lipid peroxidation rate and lipid profile in coronary heart disease. Singapore Med J 48: 415-420. 